\title{
Visualization of Irreparable Ischemic Damage in Brain by Selective Labeling of Double-Strand Blunt-Ended DNA Breaks
}

\author{
Vladimir V. Didenko, Hop Ngo, Candace L. Minchew, Denise J. Boudreaux, Marsha A. Widmayer, \\ and David S. Baskin
}

Department of Neurosurgery, Baylor College of Medicine and VAMC, Houston, TX, USA

Accepted September 15, 2002

\begin{abstract}
Background: Double-strand DNA breaks with blunt ends represent the most serious type of DNA damage, and cannot be efficiently repaired by cells. They are generated in apoptosis or necrosis and are absent in normal or transiently damaged cells. Consequently, they can be used as a molecular marker of irreparable cellular damage. We evaluated the effects of focal brain ischemia using selective labeling of blunt-ended DNA breaks as a marker of irreversible tissue damage. A new approach permitting such analysis in situ is introduced.

Materials and Methods: Rat brain sections taken 6, 24, 48 and $72 \mathrm{hr}$ after the onset of focal brain ischemia were used. Double-strand DNA breaks were detected directly in the tissue sections via ligation of blunt-ended hairpinshaped oligonucleotide probes. The probes were attached to the ends of the breaks by T4 DNA ligase. Conventional
\end{abstract}

cresyl violet co-staining and terminal transferase based labeling (TUNEL) were employed to analyze the distribution of labeled cells.

Results: Double-strand blunt-ended DNA breaks rapidly accumulate in brain cells after focal brain ischemia. At $24 \mathrm{hr}$, they concentrate in the peripheral areas of stroke, which are prone to ischemia-reoxygenation. By 48-72 hr, this type of DNA damage spreads inward, covering the internal areas of the ischemic zone.

Conclusions: Selective labeling of blunt-ended DNA breaks delineates the dynamics of stroke-induced irreversible DNA damage and provides highly specific detection of brain cells with irreparable DNA injury. It can be used for comparing the efficiency of various anti-ischemic drugs, particularly those that target DNA damage, as well as for monitoring stroke-induced damage.

\section{Introduction}

Molecular markers of cellular phenomena are important for the assessment of experimental interventions. Markers of cell death are most widely used. In cell cultures, a general assessment of cell death is performed using vital dyes, which penetrate into cells only if membrane permeability is severely disturbed. In this case, irreversible membrane damage is used as a general molecular marker of cell death. However, similar analysis is not possible in sectioned tissues, where cellular membrane integrity is compromised. Because tissue sectioning is a fundamental element of many analyses, it is important to have a general molecular marker of cell death applicable for this situation. Conventionally used terminal transferase based labeling (TUNEL) detects all possible DNA break configurations, is nonselective, and labels not only dead but also transiently damaged cells (1). In this report, we introduce detection of double-strand blunt-ended DNA breaks as a new selective marker of cell death, which is applicable for research using sectioned tissue.

Address correspondence and reprint requests to: David S. Baskin, 6560 Fannin, Suite 944, Houston, TX 77030. Phone: 713-798-4696; fax: 713-798-3227;

e-mail: dbaskin@tmh.tmc.edu
Double-strand blunt-ended DNA breaks constitute the most serious type of DNA damage. Neither DNA strand is able to provide physical integrity or information content necessary for successful repair, as can occur with single-strand breaks, where one strand in the duplex remains intact. Among various types of double-strand breaks, which include double-strand breaks with $3^{\prime}$ or $5^{\prime}$ end extensions, the blunt-ended variety represents the least efficiently repaired type. Even when ligated, these breaks still result in irreversible alteration of the DNA and cell death $(2,3)$. Generation of significant amounts of blunt-ended DNA breaks is characteristic of both apoptosis and necrosis $(4,5)$, whereas reversible cellular damage after ischemia or chemical interventions is not associated with their production (1). Other types of DNA damage, such as breaks with 3' overhangs or single-strand breaks, are either restricted to apoptotic DNA cleavage (4) or are reproduced in both transiently damaged and dying cells $(1,4)$. Thus, blunt-ended double-strand breaks represent a more specific death-associated type of DNA damage, and can serve as a biochemical common marker for cell death in both apoptosis and necrosis $(4,6)$.

Ischemia-induced cell damage produces both apoptotic and necrotic death of brain cells $(7,8)$. These processes in brain are so tightly intertwined 
that they are sometimes viewed as a single, ischemic brain-specific type of cell death (9).

In this study, we introduce a new approach to visualize irreparable stroke damage in tissue sections by detecting unrepairable double-strand bluntended DNA breaks. We use a new in situ ligation technique to detect the breaks, which serve as molecular markers of cell death. The approach exclusively labels lethally damaged cells regardless of the apoptotic or necrotic nature of their death, and provides high cell-to-cell resolution.

The assay detects blunt-ended $5^{\prime}$ phosphorylated double-strand breaks using hairpin-shaped blunt-ended oligoprobes, which are attached to the ends of the breaks by T4 DNA ligase. This permits selective detection of only a single type of DNA damage $(4,10,11)$.

Using the new approach, we demonstrate that double-strand blunt-ended DNA breaks rapidly accumulate in brain cells after focal brain ischemia, and delineate stroke-induced progression of cell death. This new approach may be useful for assessment of the efficiency of anti-ischemic therapies, particularly those that target DNA damage, as well as for monitoring stroke-induced damage.

\section{Methods}

The animal care and use portion of the following protocol was reviewed and approved by the Institutional Animal Care and Use Committee of the VA Medical Center and the Animal Protocol Review Committee of Baylor College of Medicine. All procedures were carried out with strict adherence to the protocol and the National Institutes of Health animal research guidelines.

\section{Focal Brain Ischemia}

All surgical techniques were performed under general inhalational anesthesia. Occlusion of the right middle cerebral artery (MCA) was performed in male Long Evans rats through an incision made midway between the right eye and ear. Using the operating microscope, the right MCA was exposed transcranially via a subtemporal craniotomy without damage to the zygomatic bone. Ten to twelve millimeters of the MCA, beginning ventral to the olfactory tract, was permanently occluded using bipolar coagulation.

The animals were transcranially perfused with paraformaldehyde $6,24,48$, and $72 \mathrm{hr}$ after occlusion, and the brains were removed and post-fixed overnight. A 2-mm thick coronal block was cut from the center of the MCA territory, beginning $\sim 0.5 \mathrm{~mm}$ anterior to the optic chiasm and ending $\sim 0.5 \mathrm{~mm}$ posterior to the optic chiasm. The tissue was embedded in paraffin and cut in $6-\mu \mathrm{m}$ thick sections. Sections used in the current study were taken from the middle area of the tissue block. Each staining condition was repeated in four separate sections from each of the eight animals.

\section{Cresyl Violet Staining}

The extent of the ischemic injury was determined by cresyl violet staining. The sections were deparaffinized with xylene, dehydrated in $100 \%$ alcohol $(3 \times 2 \mathrm{~min})$ and stained in $0.02 \%$ cresyl violet solution for $60 \mathrm{~min}$. They were briefly washed in $95 \%$ and $100 \%$ ethanol, cleared in xylene, and covered with Permount. The area of cellular damage was clearly identified using this technique.

\section{The Oligoprobe for In Situ Ligation}

The oligoprobe sequence was designed to avoid misfolding into any structure other than a blunt-ended hairpin, so that it was able to ligate to blunt-ended DNA breaks. Biotin was incorporated into the probes by chemical insertion of biotin triethylene glycol (TEG) phosphoramidite (Glen Research, Sterling, VA, USA) directly into the oligonucleotide backbone (Intergen Company, Purchase, NY, USA). The sequence of the probe was: $5^{\prime}$ GCG CTA GAC C $5^{*} \mathrm{G}$ GTC TAG CGC $3^{\prime}$ (5*- represents biotin-TEG spacer). Probes were detected in a reaction with a streptavidin-FITC conjugate (Vector Laboratories, Burlingame, CA, USA) (see In Situ Ligation).

\section{In Situ Ligation}

In situ ligation using hairpin shaped oligoprobes was performed as previously described (9). Briefly, $6-\mu \mathrm{m}$ thick sections were deparaffinized with xylene, rehydrated in graded alcohol concentrations, washed in water, and treated with proteinase $\mathrm{K}$ $(50 \mu \mathrm{g} / \mathrm{ml})$ for $15 \mathrm{~min}$. Sections were rinsed with water and incubated in $80 \mu \mathrm{l}$ of the ligation buffer without the probe $(66 \mathrm{mM}$-Tris $\mathrm{HCl}, \mathrm{pH} 7.5,5 \mathrm{mM}$ $\mathrm{MgCl}_{2}, 0.1 \mathrm{mM}$ dithioerythritol, $1 \mathrm{mM}$ ATP, and $15 \%$ polyethylene glycol-8000) for $15 \mathrm{~min}$ to ensure even saturation. The buffer was aspirated, and the full ligation mix containing the ligation buffer with the hairpin probe $(35 \mu \mathrm{g} / \mathrm{ml})$ and T4 DNA ligase $(250 \mathrm{U} / \mathrm{ml})$ was applied to the sections. As a mock control reaction, an equal volume of $50 \%$ glycerol in water was substituted for T4 DNA ligase.

Sections were incubated in a humidified chamber for $16 \mathrm{hr}$ at $23^{\circ} \mathrm{C}$, and were then briefly washed in water. The signal was visualized using TSA-direct kit (NEN Life Science Products, Boston, MA, USA), with color development stopped on all sections simultaneously. Sections were then counterstained with 4,6-diamidino-2-phenylindole ( $1 \mu \mathrm{g} / \mathrm{ml})$.

\section{Terminal Transferase Based Nick-End Labeling}

TUNEL staining for detection of free $3^{\prime} \mathrm{OH}$ groups in cellular DNA was employed in a dual labeling reaction with in situ ligation. The in situ ligation procedure was finished first and was followed by TUNEL, which was performed using the ApoTaq Red In Situ Apoptosis Detection kit with rhodamine (Serologicals, Gaithersburg, MD, USA), with the standard technique recommended by the manufacturer. 


\section{Results}

Figure 1 represents the principle of the assay for selective in situ labeling of blunt-ended DNA breaks. It demonstrates a double-strand oligonucleotide probe with blunt ends ligating to a genomic bluntended DNA break. The ligation occurs if a break possesses a $5^{\prime} \mathrm{PO}_{4}$ group, needed for the creation of a bond by T4 DNA ligase. The $5^{\prime}$ phosphates at the ends of the breaks are either produced in the cleavage reaction by apoptotic or nonspecific cellular nucleases, or are introduced by kinases and transferases acting on $5^{\prime} \mathrm{OH}$ bearing DNA ends generated by the lysosomal DNase II.

To make a rough estimate of the sensitivity of the assay in our conditions, in preliminary experiments we established that our detection system composed of an Olympus IX-70 microscope with an attached MicroMax digital videocamera, can visualize 1.25 fmole of FITC spotted as a $1.3 \mathrm{~mm}$ dot (not shown). This corresponds to $\sim 45,000$ FITC molecules per $80 \mu \mathrm{m}^{2}$, the area of a $10-\mu \mathrm{m}$ diameter nucleus. Using a TSA-direct kit for fluorescence development, the signal can be increased by two orders of magnitude. As the assay places a single biotin at the end of each break, detection of several thousand double-strand blunt-ended breaks per nucleus is observable. This number of breaks far exceeds the repair capabilities of any cell, and is comparable to the total number of breaks observed in the initial stages of apoptosis (12).

Figure 2 illustrates the dynamics of the irreparable brain tissue damage at different time-points after the onset of focal brain ischemia in rat, as visualized by labeled cells with high concentrations of bluntended DNA breaks (upper panel; green fluorescence). A traditional cresyl violet staining is provided for comparison (lower panel).

After $6 \mathrm{hr}$ of ischemia, the boundary of the ischemic zone is already clearly delineated by the cells with extensive DNA damage labeled by in situ ligation (Fig. 2A) and relatively small numbers of

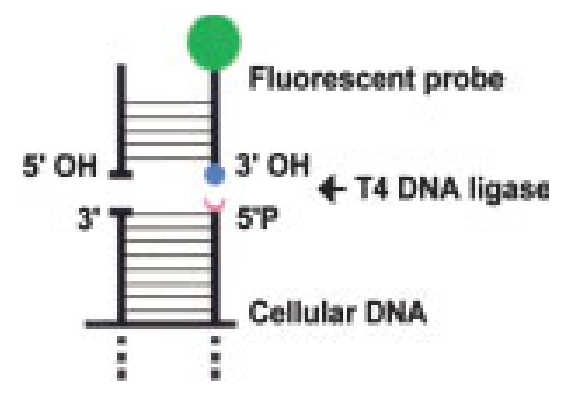

Fig. 1. Schematics of in situ ligation for selective detection of blunt-ended DNA breaks in tissue sections. The blunt-ended probe, shown as a double-strand DNA fragment carrying a single fluorophore, is attached to the end of a blunt-ended DNA break in tissue section using T4 DNA ligase. The detected break possesses $5^{\prime}$ phosphate, needed for the ligation reaction. irreversibly damaged cells are located scattered throughout the zone. After $24 \mathrm{hr}$ of focal brain ischemia, cell death and DNA injury are much more pronounced (Fig. 2B). Interestingly, at $24 \mathrm{hr}$, the dying cells with extensive double-strand blunt-ended DNA breaks are concentrated at the boundaries of the stroke. The signal is particularly strong in many areas neighboring undamaged tissue. This suggests that ischemia-reoxygenation episodes may contribute to the distribution of DNA damage and cell death. These reoxygenation episodes frequently occur at the boundary between normal and ischemic tissue.

After $48 \mathrm{hr}$ of continuing ischemia, the progression of cell death results in the appearance of large numbers of dying cells in the central parts of the ischemic zone. Seventy-two hours after ischemia onset, cells with high numbers of double-strand blunt-ended DNA breaks occupy all parts of the ischemic zone.

The comparison of in situ ligation-based assessment of brain damage with cresyl violet staining illustrates the much higher sensitivity of the new approach, especially at the earliest time points. Because the new approach is able to resolve the signal at a single cell level, it permits both a general overview of the damaged area, as well as an analysis of cellular populations affected by ischemia (Fig. 2).

The general territory occupied by stroke at $72 \mathrm{hr}$ is already outlined at $6 \mathrm{hr}$ by in situ ligation, but not with cresyl violet staining. The cresyl violet staining does not show the dynamics of stroke progression in comparable detail to the new approach, which clearly visualizes several stages.

Figure 3 demonstrates the early generation of double-strand breaks at the boundaries of stroke, and the subsequent progression of their generation and cell death inward into the ischemic zone. This early cell death at the boundary of stroke can be explained by the repeated episodes of ischemia-reoxygenation, which have been shown to occur in the peripheral areas of brain ischemia on the boundary with unaffected tissue and can worsen cellular damage in these areas via production of free radicals (13).

Interestingly, in areas not bordering healthy tissue, the in situ ligation signal can appear at a later time compared to the internal brain areas (Fig. 4). Colabeling using TUNEL demonstrates that ischemiadamaged cells in this area can initially become TUNEL positive, and then later show the in situ ligation signal (Fig. 4).

\section{Discussion}

The detection of blunt-ended double-strand DNA breaks represents a new modality useful for determining the extent and distribution of irreversible cellular damage and death in ischemic brain. This type of break is associated with lethal injury, thus transiently damaged cells are not labeled. In previous reports, we describe the use of in situ ligation for detection of 

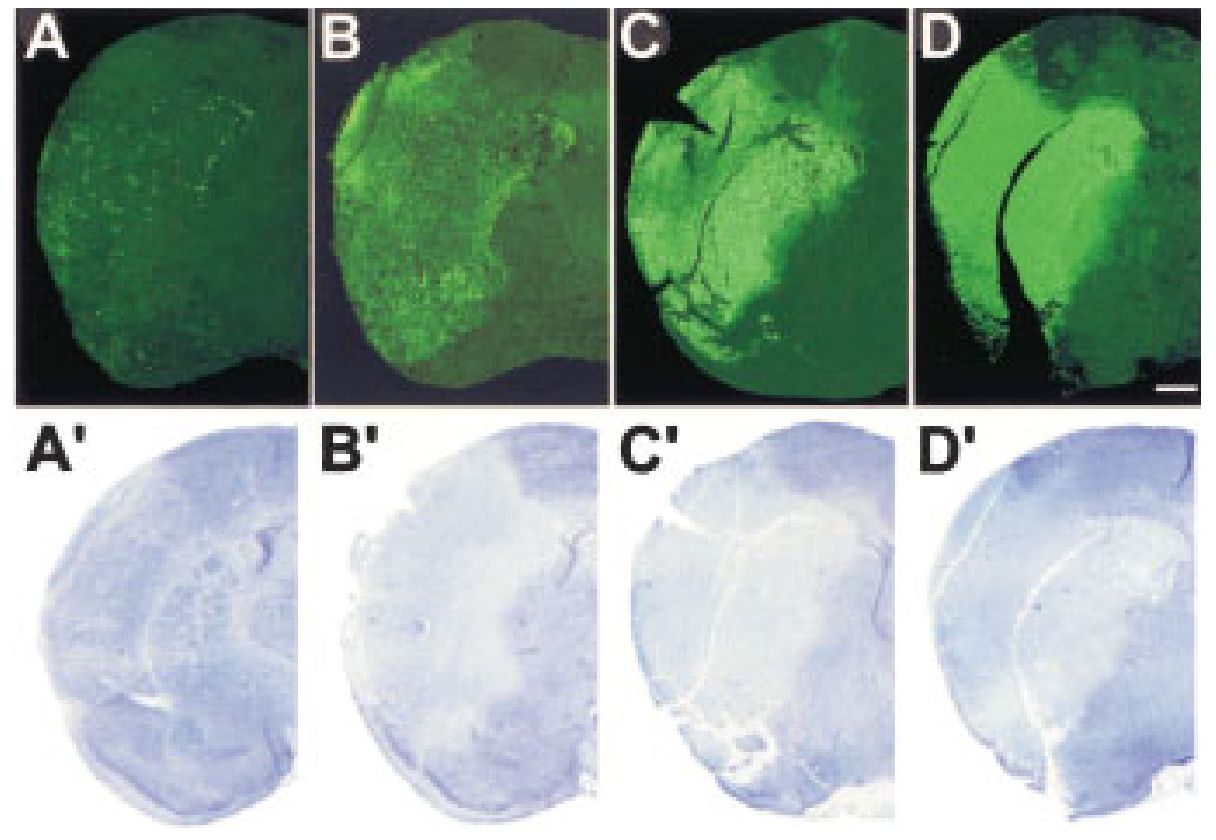

specific DNA breaks created by the apoptotic nucleases, with particular emphasis on detection of short $3^{\prime}$ overhangs $(4,10,11)$. In this paper, however, attention is shifted to detection of blunt-ended breaks, which expands the utility of the approach.

Blunt-ended DNA breaks are not found in normal brain cells. Very small numbers of blunt-ended breaks (one or several/cell) can occur during early Band T-cell development in a process of $\mathrm{V}(\mathrm{D}) \mathrm{J}$ recombination (3). However the detection of these single breaks is below the limit of sensitivity of the in situ ligation technique (4) and, therefore, will not produce false positives.

Several other labeling techniques are currently employed for the determination of cell injury in brain ischemia. Cresyl violet staining is traditionally used for visualization of stroke in tissue sections. The technique labels damaged areas via negative staining, because the injured cells do not retain the dye. Being simple, it is frequently used. However, the absence of detection of an identifiable molecular marker of damage makes the technique less appealing. In addition, its ability to visualize stroke damage, especially

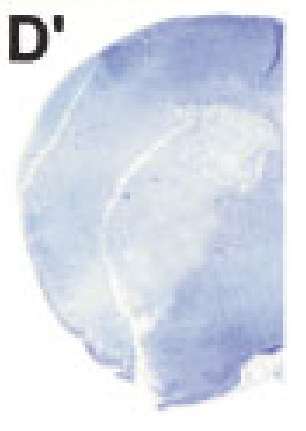
approach (Fig. 2).
Fig. 2. Comparison of in situ ligation and cresyl violet staining for the detection of stroke induced brain damage. (A, B, C, D) In situ ligation based detection of blunt-ended doublestrand DNA breaks. (A', B', $\left.\mathbf{C}^{\prime}, \mathbf{D}^{\prime}\right)$ Cresyl violet staining $6 \mathrm{hr}$ after onset of focal ischemia $\left(\mathrm{A}^{\prime}\right), 24 \mathrm{hr}$ after onset of focal ischemia $\left(\mathrm{B}^{\prime}\right), 48 \mathrm{hr}$ after onset of focal ischemia $\left(C^{\prime}\right)$, and $72 \mathrm{hr}$ after onset of focal ischemia $\left(D^{\prime}\right)$. Note the precise delineation of cellular damage on a cell-by-cell basis with the in situ ligation technique, and the lack of said precision using cresyl violet. (Bar, $850 \mu \mathrm{m}$ ).

early, is much less specific compared to the new

Terminal transferase labeling (TUNEL staining) is also often employed for visualization of cell damage in stroke. Yet the method indiscriminately detects any exposed $3^{\prime} \mathrm{OH}$ groups, some of which can be present in transiently damaged (1) and even normal dividing cells (14). In addition it is prone to artifactual labeling, as single-strand breaks of various kinds can be created in tissue fixation (15) and processing such as microwave treatment in costaining with antibodies (4) and even in the process of cutting sections by microtome (16). Assessment of the effectiveness of anti-apoptotic interventions using TUNEL is difficult due to the fact that surviving cells often still contain high numbers of reparable single-strand DNA breaks $(1,17)$.

Although the in situ ligation signal appears several hours later compared to the TUNEL signal, it is far less ambiguous and has more value in comparing the effects of different interventions, because in such assessments, it is critical to discriminate between lethally and nonlethally damaged cells.
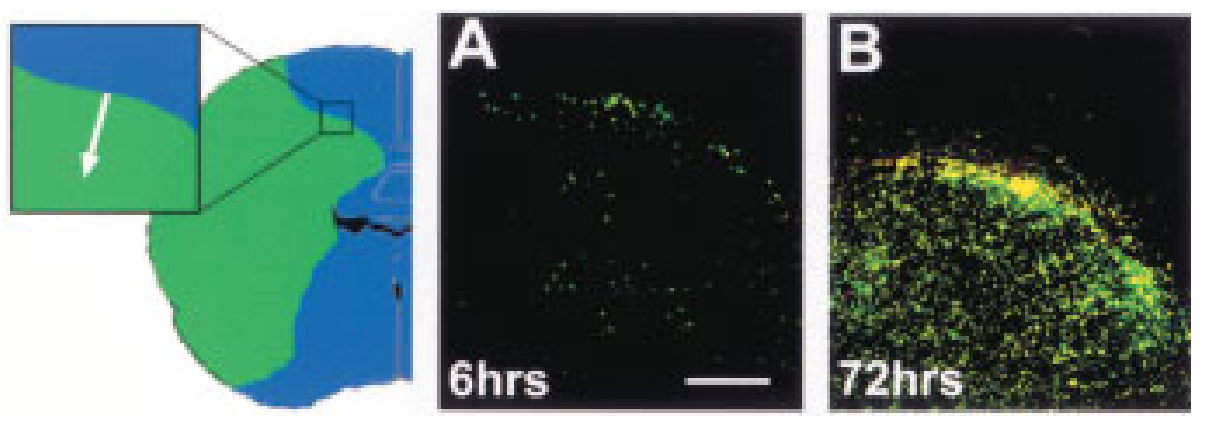

Fig. 3. Detection of lethally damaged cells at the boundary of stroke by in situ ligation at 6 and $72 \mathrm{hr}$ after the onset of ischemia. The first dying cells appear at the boundary at the earliest time point of $6 \mathrm{hr}$. Large numbers of dying cells are demonstrated within the stroke area at a later time of $72 \mathrm{hr}$ post-ischemia. The arrow in the inset indicates the direction of progression of DNA damage and cell death as time goes by. (Bar, $150 \mu \mathrm{m}$ ). 

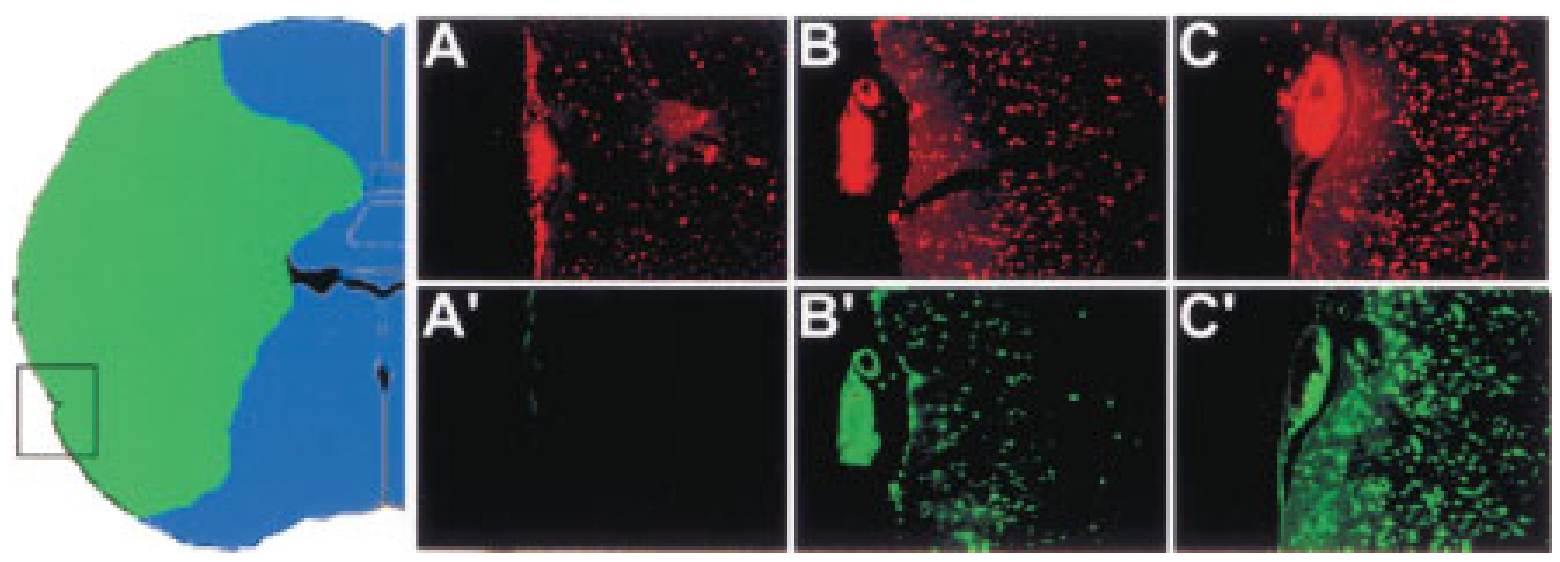

Fig. 4. Dual detection of DNA breaks using TUNEL $(A, B, C)$ and in situ ligation $\left(A^{\prime}, B^{\prime}, C^{\prime}\right)$. Images are from 6 hr $\left(A, A^{\prime}\right)$, $24 \mathrm{hr}\left(\mathrm{B}, \mathrm{B}^{\prime}\right)$, and $72 \mathrm{hr}\left(\mathrm{C}, \mathrm{C}^{\prime}\right)$ after ischemia onset. DNA damage starts with detection of a variety of breaks detectable by TUNEL at $6 \mathrm{hr}$ after ischemia onset and gradually proceeds toward lethal DNA damage at later times of ischemia (24 and $72 \mathrm{hr})$. The boxed area indicates the general area from which the images were taken (Bar, $100 \mu \mathrm{m}$ ).

The new approach detects irreversible tissue damage only when it is accompanied by extensive DNA degradation, which occurs in ischemia, reoxygenation, apoptosis, radiation exposure, and anticancer treatment with DNA-targeting drugs. In some other situations, such as instant cell death caused by chemical agents disrupting cellular membranes or by rapid freezing, cell death is not accompanied by extensive DNA damage. We believe that the major utility of this new approach is in assessing the efficiency of various anti-ischemic, antineoplastic, and antiradiation interventions, where it could provide valuable quantitative comparisons of the extent of irreversible DNA damage.

Detection of a marker for lethal cell injury is also important in the analysis of ischemic brain tissue. The absence, or considerable variability, of the classical markers of apoptotic cell death was demonstrated in ischemic brain. For example, different sets of activated caspases were detected in various apoptotic brain cells (18). In addition, the absence of classical apoptotic morphology has recently been demonstrated in a number of apoptotic brain cells (19). It is also important that the necrotic component of cell injury cannot be selectively labeled; its detection requires morphologic evaluation on a cell-by-cell basis (19).

Because in situ ligation detects an important and early point of no return for lethal cell injury of all types, we believe that it will be of value for delineation of lethal DNA damage in brain tissue. It is not intended to replace traditional staining techniques used to delineate infarct size, such as cresyl violet and TTC. Unlike other available techniques, it focuses on a parameter of paramount importance, namely, a molecular marker of lethal cell injury.

\section{Acknowledgments}

This research was supported by grant R01 CA78912 from the National Cancer Institute, National Insti- tutes of Health (D.S.B.), by grant 004949-054 from the Texas Higher Education Coordinating Board (D.S.B. and V.V.D.), and by grants from DeBakey Medical Foundation (V.V.D.), Baylor College of Medicine (V.V.D.), The Taub Foundation, The Henry J.N. Taub Fund for Neurosurgical Research, The George A. Robinson, IV Foundation, The Blanche Greene Estate Fund of The Pauline Sterne Wolff Memorial Foundation, and The Seigo Arai and Koppelman Funds of The Neurological Research Foundation (D.S.B.).

\section{References}

1. Didenko VV, Wang X, Yang L, Hornsby PJ. (1999) DNA damage and p21WAF1/CIP1/SDIl in experimental injury of the rat adrenal cortex and trauma-associated damage of the human adrenal cortex. J. Pathol. 189: 119-126.

2. Lips J, Kaina B. (2001) DNA double-strand breaks trigger apoptosis in p53-deficient fibroblasts. Carcinogenesis 22: 579585.

3. Lieber MR. (1998) Pathological and physiological doublestrand breaks: roles in cancer, aging, and the immune system. Am. J. Pathol. 153: 1323-1332.

4. Didenko VV, Hornsby PJ. (1996) Presence of double-strand breaks with single-base $3^{\prime}$ overhangs in cells undergoing apoptosis but not necrosis. J. Cell Biol. 135: 1369-1376.

5. Al-Lamki RS, Skepper JN, Loke YW, et al. (1998) Apoptosis in the early human placental bed and its discrimination from necrosis using the in-situ DNA ligation technique. Hum. Reprod. 13: 3511-3519.

6. Al-Lamki RA, Skepper JN, Loke YW, et al. (1998) Apoptosis in the early human placental bed and its discrimination from necrosis using the in situ DNA ligation technique. Hum. Reprod. 13: 3511-3519.

7. Morita-Fujimura Y, Fujimura M, Yoshimoto T, Chan PH. (2001) Superoxide during reperfusion contributes to caspase8 expression and apoptosis after transient focal stroke. Stroke 32: 2356-2361.

8. Moroni F, Meli E, Peruginelli F, et al. (2001) Poly(ADPribose) polymerase inhibitors attenuate necrotic but not apoptotic neuronal death in experimental models of cerebral ischemia. Cell Death Differ. 8: 92 1-932.

9. Fukuda T, Wang H, Nakanishi H, et al. (1999) Novel nonapoptotic morphological changes in neurons of the mouse 
hippocampus following transient hypoxic-ischemia. Neurosci. Res. 33: 49-55.

10. Didenko VV, Tunstead JR, Hornsby PJ. (1998) Biotin-labeled hairpin oligonucleotides: probes to detect double-strand breaks in DNA in apoptotic cells. Am. J. Pathol. 152: 897-902.

11. Didenko VV, Boudreaux DJ, Baskin DS. (1999) Substantial background reduction in ligase-based apoptosis detection using newly designed hairpin oligonucleotide probes. Biotechniques 27: 1130-1132.

12. Walker PR, Leblanc J, Carson C, et al. (1999) Neither caspase-3 nor DNA fragmentation factor is required for high molecular weight DNA degradation in apoptosis. Ann. N.Y. Acad. Sci. 887: 48-59.

13. Kontos HA. (2001) Oxygen radicals in cerebral ischemia: the 2001 willis lecture. Stroke 32: 2712-2716.

14. Li X, Darzynkiewicz Z. (1995) Labelling DNA strand breaks with BrdUTP. Detection of apoptosis and cell proliferation. Cell Prolif. 28: 571-579.
15. Grasl-Kraupp B, Ruttkay-Nedecky B, Koudelka H, et al. (1995) In situ detection of fragmented DNA (TUNEL assay) fails to discriminate among apoptosis, necrosis, and autolytic cell death: a cautionary note. Hepatology 21 : 1465-1468.

16. Sloop GD, Roa JC, Delgado AG, et al. (1999) Histologic sectioning produces TUNEL reactivity. A potential cause of falsepositive staining. Arch. Pathol. Lab. Med. 123: 529-532.

17. Dikomey E, Lorenzen J. (1993) Saturated and unsaturated repair of DNA strand breaks in CHO cells after X-irradiation with doses ranging from 3 to 90 Gy. Int. J. Radiat. Biol. 64: 659-667.

18. Velier JJ, Ellison JA, Kikly KK, et al. (1999) Caspase-8 and caspase- 3 are expressed by different populations of cortical neurons undergoing delayed cell death after focal stroke in the rat. J. Neurosci. 19: 5932-5941.

19. Martin LJ, Al-Abdulla NA, Brambrink AM, et al. (1998) Neurodegeneration in excitotoxicity, global cerebral ischemia and target deprivation: a perspective on the contributions of apoptosis and necrosis. Brain Res. Bull. 46: 281-309. 